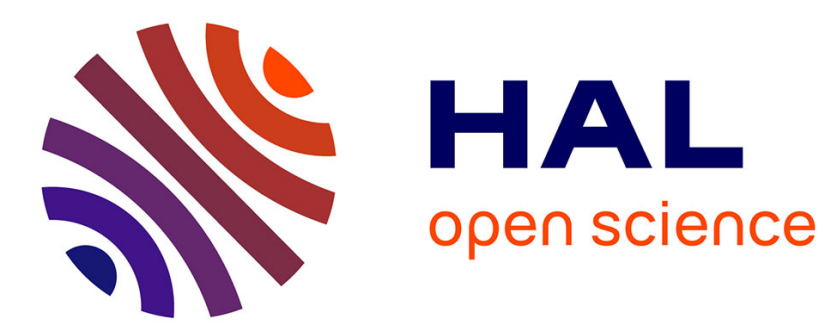

\title{
A new Monte Carlo tool for organ dose estimation in computed tomography
}

\author{
Camille Adrien, Cindy Le Loirec, S. Dreuil, Jean-Marc Bordy
}

\section{To cite this version:}

Camille Adrien, Cindy Le Loirec, S. Dreuil, Jean-Marc Bordy. A new Monte Carlo tool for organ dose estimation in computed tomography. Radioprotection, In press, 55 (2), pp.123-134. 10.1051/radiopro/2020006 . cea-03038511

\section{HAL Id: cea-03038511 https://hal-cea.archives-ouvertes.fr/cea-03038511}

Submitted on 1 Oct 2021

HAL is a multi-disciplinary open access archive for the deposit and dissemination of scientific research documents, whether they are published or not. The documents may come from teaching and research institutions in France or abroad, or from public or private research centers.
L'archive ouverte pluridisciplinaire HAL, est destinée au dépôt et à la diffusion de documents scientifiques de niveau recherche, publiés ou non, émanant des établissements d'enseignement et de recherche français ou étrangers, des laboratoires publics ou privés. 


\section{A new Monte Carlo tool for organ dose estimation in}

\section{Computed Tomography}

3 Camille Adrien ${ }^{1}$, Cindy Le Loirec ${ }^{1 *}$, Serge Dreuil ${ }^{2}$, Jean-Marc Bordy ${ }^{1}$

$4 \quad{ }^{1}$ CEA, List, PC 181, CEA-Saclay 91191 Gif-sur-Yvette Cedex, France

$5 \quad{ }^{2}$ Service de physique médicale, Gustave Roussy, 94805 Villejuif, France

6 * Cindy.LELOIREC@cea.fr

7 Abstract (200 words):

8 The constant increase of computed tomography (CT) exams and their major contribution to the

9 collective dose led to international concerns regarding patient dose in CT imaging. Efforts were

10 made to manage radiation dose in $\mathrm{CT}$, mostly with the use of the CT dose index (CTDI). However

11 CTDI does not give access to organ dose information, while Monte Carlo (MC) simulation can

12 provide it if detailed information of the patient anatomy and the source are available.

13 In this work the X-ray source and the geometry of the GE VCT Lightspeed 64 were modelled,

14 based both on the manufacturer technical note and some experimental data. Simulated dose

15 values were compared with measurements performed in homogeneous conditions with a pencil

16 chamber and then in CIRS ATOM anthropomorphic phantom using both optically stimulated

17 luminescence dosimeters (OSLD) for point doses and XR-QA Gafchromic $\circledast$ films for relative dose

18 maps. Organ doses were ultimately estimated in the ICRP 110 numerical female phantom and

19 compared to data reported in the literature.

20 Comparison of measured and simulated values show that our tool can be used for a patient

21 specific and organ dose oriented radiation protection tool in CT medical imaging.

22 Keywords: Monte Carlo Simulation, Computed Tomography, Organ dose 
231 Introduction

24 Due to the significant rise of computed tomography (CT) exams in the past few years and the 25 resulting increase of the collective dose (Kalender 2014), patient dose in CT imaging has become 26 a subject of interest in public health, especially for children (Akhlaghi et al 2015, Journy et al 27 2017a, Journy et al 2017b, Habib et al 2019). Considerable efforts have thus been made these 28 past few years to manage radiation dose in CT (Coakley et al 2010, Amis 2011, Sodickson 2012 29 and Mayo-Smith et al 2014). However CT protocols are still traditionally optimized using the CT 30 dose index (CTDI), which is not representative of the patient dose (Kalender 2014 and McCollough 31 et al 2011). Patient-specific dose reports, including absorbed dose to organs, should thus be 32 suitable for individualized protocol optimization. Because absorbed dose to organ cannot be 33 directly measured, some research groups attempt to adapt the treatment planning system (TPS) 34 used in radiation therapy for kV x-ray beams dosimetry. For example, Alaei et al 2000 investigated 35 the accuracy of a convolution/superposition TPS for predicting kV beam dosimetry, but they 36 reported dose discrepancies up to $145 \%$ in the region surrounding bone heterogeneities. Others 37 groups developed specific software based on precomputed Monte Carlo (MC) data, such as CT 38 imaging $^{1}$ (Kalender et al 1999) and ImPact $^{2}$. Axial or helical protocols are included in these 39 software, but large discrepancies can occur for helical acquisitions, since doses are approximated 40 from contiguous axial scan. Instead of approximating dose from precomputed MC data, other

41 research groups developed their own $M C$ software to directly estimate organ dose using 42 computational patient models or patient DICOM images as inputs.

43 Most of the time, code benchmarking was performed using either a CTDI phantom 44 (Jarry et al 2003, De Marco et al 2005 and Deak et al 2008) or a custom-designed cylindrical 
45 phantom which enables dose measurements at seven different radial distances from its central 46 axis ( $\mathrm{Li}$ et al 2011). That approach has some limitations because standard and custom-made 47 phantoms are made of PMMA and only permit dose measurements at selected distances from the 48 central axis. Impact of heterogeneities and 2D dose gradients cannot be estimated with such 49 phantoms. Due to the limitations of validation in homogeneous conditions, benchmark in 50 anthropomorphic conditions is mandatory. For that purpose, DeMarco et al 2005 put MOSFET 51 detectors on the surface of a male anthropomorphic phantom, while Deak et al 2008, as well as 52 Li et al 2011, choose to insert TLD (ThermoLuminescent Dosimeter) chips into Alderson-Rando 53 phantom.

54 In this work we present the dosimetric validation of a MC tool based on PENELOPE 55 (Sempau et al 2003) by comparing simulated and experimental dose estimations. Without any 56 detailed information provided by the manufacturer, the CT scanner was first modelled only using 57 information provided by the manufacturer technical note and the method proposed by 58 Turner et al 2009. In a first step the model has been validated in homogeneous conditions by 59 comparing experimental and simulated integrated dose obtained with a pencil chamber. Simulated 60 and measured CTDI values have also been compared. Then point dose measurements in an 61 anthropomorphic phantom, using optically stimulated luminescence detectors (OSLD), were 62 compared with simulated dose values. Dose distributions in the phantom were also measured with

63 Gafchromic XR-QA2 films and compared with the simulated dose distributions to validate the 64 calculated dose gradient in anthropomorphic conditions.

65 Although anthropomorphic validations are mandatory, they require a detector with weak energy 66 dependence and an accurate calibration for the energy spectra involved in CT. Such conditions 67 are not met nowadays with ionization chamber used in CT. Calibrations are usually performed for 68 one normalized spectrum with uncertainties higher than 3-5\% and without any correction factor 69 for other beam qualities. Therefore, given the high number of parameters influencing the 
70 measurements and their high uncertainties (about 10\% for device parameters such as mAs), 20\%-

71 differences between simulated and measured results are generally considered as a good

72 approximation of the real situation and were chosen in the present study as the success criterion.

73 Finally, as an application case of the tool, we estimated organ doses with a numerical

74 anthropomorphic phantom.

\section{Monte Carlo simulation}

\section{$76 \quad 2.1 \quad$ CT scanner}

77 The VCT Lightspeed 64 CT scanner (GE Healthcare, Waukesha, WI) is a third-generation

78 scanner. As specified in the technical note, focal spot to isocentre distance is $541 \mathrm{~mm}$. Target is

79 a tungsten-rhenium alloy with a 7 degree tilt angle regarding to reference axis. Dual focal spots

80 are used depending on current and voltage values. According to IEC 60336, small focal spot is

$810.7 \mathrm{~mm} \times 0.6 \mathrm{~mm}$ and large focal spot is $0.9 \mathrm{~mm} \times 0.9 \mathrm{~mm}$. The beam full width at half maximum

82 (FWHM) is adapted as a function of the focal spot size and the chosen aperture. For a $40 \mathrm{~mm}$

83 beam aperture, the FWHM are 42.6 and $42.9 \mathrm{~mm}$ for the small and the large focal spots,

84 respectively. Use of bowtie filter is related to a maximum Scan Field of View (SFOV). The

85 maximum SFOV is $32 \mathrm{~cm}$ for the small bowtie filter reported as "Ped Body" and $50 \mathrm{~cm}$ for the large

86 bowtie filter reported as "Large Body". Two tube potentials (100 and $120 \mathrm{kVp}$ ), two bowtie filters

87 ("Ped Body" and "Large Body") and a $40 \mathrm{~mm}$ beam collimation are modelled and presented in this 88 work.

$89 \quad 2.2 \quad \underline{\text { CT model }}$

90 The 2006 release of the MC code PENELOPE (Sempau et al 2003) is used to model the VCT

91 Lightspeed 64 CT scanner (GE Healthcare, Waukesha, WI).

92 For MC simulations, all elements of the X-ray tube need to be modelled. Without any detailed

93 information provided by the manufacturer, filtration elements are modelled by adapting the 
94 experimental method described by Turner et al 2009. Based on an initial soft spectrum and

95 experimental data, this method provides information on equivalent inherent filtration and 96 equivalent bowtie filter shapes, which reproduce the attenuation of the real filtration elements.

97 These filtration elements are reported in table 1. For the bowtie filter only the central filtration is 98 indicated but the complete equivalent shape is taken into account in the simulation.

Table 1: Filtration of the studied beams.

\begin{tabular}{ccc}
\hline \hline Potential (kVp) & SFOV $(\mathrm{cm})$ & Total filtration (inherent + central bowtie filtration). \\
\hline 100 & 50 & $5.20 \mathrm{~mm} \mathrm{Al}$ \\
120 & & $4.65 \mathrm{~mm} \mathrm{Al}$ \\
100 & 32 & $3.44 \mathrm{~mm} \mathrm{Al}$ \\
120 & & $2.89 \mathrm{~mm} \mathrm{Al}$
\end{tabular}

100 One of the specificities of CT scanner is the tube motion of the gantry during the acquisition. Both 101 scanning modes, axial and helical, associated with a specific tube path, has to be carefully 102 implemented into the MC tool. For that purpose a specific source is defined. Instead of using a 103 direct sampling of particles along the tube path and a limited particle splitting (Li et al 2011), it was 104 taken advantage of the symmetries in geometry to implement a pipe shaped source with two 105 variance reduction techniques: a circular splitting and a translational one.

106 The different elements of the X-ray tube are modeled with PENELOPE according to the 107 manufacturer technical note ${ }^{3}$ and to Turner method (Turner et al 2009) for the filtration. A PSF is 108 created below the bowtie filter at $15 \mathrm{~cm}$ from the focal spot (figure 1). This PSF contains all the 
110 weight, particle type. Each stored particle is read, split and released for simulation.

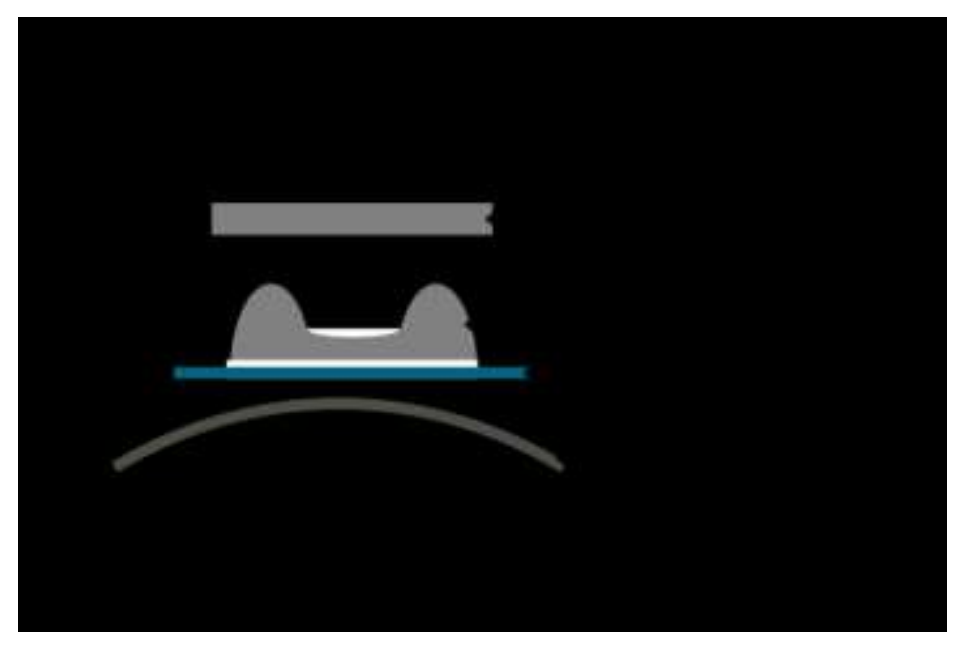

(a) CT scanner head and PSF position
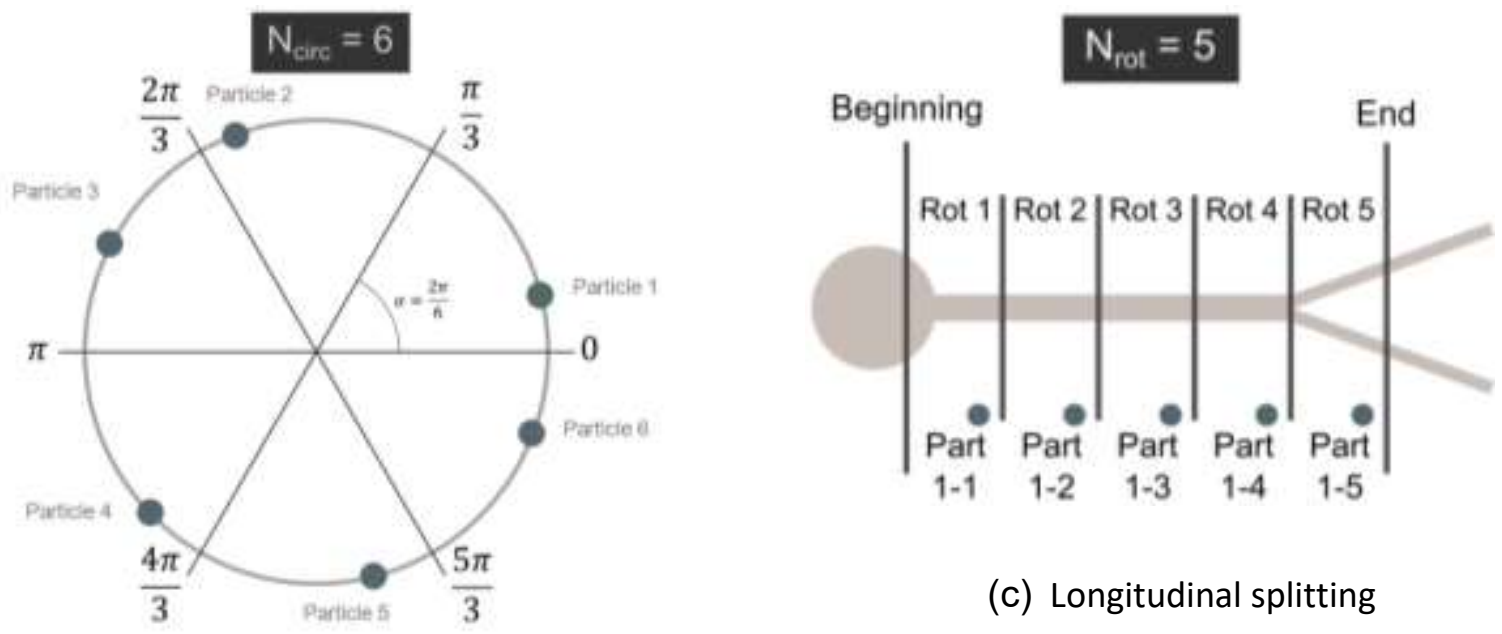

(b) Circular splitting

111 Figure 1: Information about the CT geometry (a), the PSF location (a), the circular splitting (b) and the longitudinal splitting (c).

113 For the circular splitting, the initial particle is splitted in $N_{C}$ particles. Each splitted particle is

114 sampled on an arc of circle by a random angle $(\varphi)$ in the interval $\left[2 \pi \times i / N_{C}: 2 \pi \times(i+1) / N_{C}\right]$ 
115 with $i$ between 0 and $\left(N_{C}-1\right)$. User can define $N_{C}$ knowing that a large $N_{C}$ improves the statistics

116 but increases the computation time and might introduce bias in the simulation results. For both

117 scanning modes $X$ and $Y$ particle coordinates are modified, with $X$ and $Y$ axes the transverse and

118 vertical directions, respectively. In axial mode simulation, the $Z$ coordinate remains unchanged.

119 On the contrary, for helical acquisition, the $Z$ coordinate is modified according to equation 1 :

$$
z^{\prime}=z+\varphi / 2 \pi \times \text { pitch } \times \text { collimation }(e q u .1)
$$

121 Once the circular splitting is realized, each particle is splitted a second time in $N_{R}$ particles for the

122 translational splitting. The $N_{R}$ value is determined according to the acquisition parameters as:

$$
N_{R}=\frac{\text { total explored length }}{\text { pitch } \times \text { collimation }}(\text { equ.2) }
$$

124 so that it allows a complete covering of the scanning range along the $Z$ axis. The new $Z$ coordinate 125 is then determined according to equation 3, placing each $N_{R}$ particle on a gantry rotation.

$$
z^{\prime \prime}=z^{\prime}+i \times \text { pitch } \times \text { collimation with } i \in\left[0, N_{R}\right](e q u .3)
$$

127 Due to the lack of information regarding the tube starting position, which is known to largely

128 influence dose results (Zhang et al 2009), a unique tube path cannot be determined. Choosing 129 only one tube path can undeniably lead to a dose underestimation/overestimation for some 130 peripheral organs if the real tube motion is largely different of the simulated path. Because a dose 131 underestimation cannot be considered as this tool is designed for radiation protection purposes, 132 choice is made to simulate every possible path. In such a way dose is systematically 133 overestimated because all peripheral organs will "see" the tube along its path. Tube starting angle 134 is thus randomly chosen for each initial particle.

\section{$135 \quad 2.3 \quad$ Analysis of MC data}

136 The tube loading information is used to normalize MC results. The tube loading can be related to 137 a number of emitted electrons $\left(N_{e}\right)$ according to equation 4 , where I represents the CT scanning 
138 current expressed in Ampere, $t$ the acquisition time expressed in second, and $e$ the electron 139 elementary charge expressed in Coulomb.

$$
N_{e}=\frac{I(A) \times t(s)}{e}(e q u .4)
$$

141 Emitted primary electrons are actually related to the simulated primary showers in the MC 142 simulation.

\section{$143 \quad 2.3 .1 \quad$ Deposited energy in a volume}

144 To validate the MC model in homogeneous conditions a RadCal 10X6-3CT pencil chamber (RTI

145 electronics) is used and modeled as a $10 \mathrm{~cm}$ length cylinder. The cylinder radius is the one

146 reported in the chamber documentation. The parameters used for the simulations are reported in

147 table 2. Elastic-scattering parameters $C_{1}$ and $C_{2}$ are set to 0.05 to have simulations with the

148 highest precision. Values of the cut-off energies $W_{C C}$ and $W_{C R}$ are fixed at $100 \mathrm{eV}$. Absorption

149 energies of $100 \mathrm{keV}$ for electrons and positrons is compatible with the volume of detection as the 150 range of a $100 \mathrm{keV}$ electron is about $0.15 \mathrm{~mm}$ in water.

Table 2: MC simulation parameters used for the homogeneous acquisitions.

\begin{tabular}{llllllll}
\hline Material & $E_{\mathrm{abs}, \mathrm{e}-}$ & $\mathrm{E}_{\mathrm{abs}, \text { photons }}(\mathrm{eV})$ & $\mathrm{E}_{\mathrm{abs}, \mathrm{e}+}$ & $\mathrm{C} 1$ & $\mathrm{C} 2$ & $\mathrm{~W}_{\mathrm{cc}}(\mathrm{eV})$ & $\mathrm{W}_{\mathrm{CR}}(\mathrm{eV})$ \\
& $(\mathrm{eV})$ & & $(\mathrm{eV})$ & & & & \\
& $10^{3}$ & $10^{2}$ & $10^{4}$ & 0.05 & 0.05 & $10^{2}$ & $10^{2}$ \\
Air & $10^{3}$ & $10^{2}$ & $10^{4}$ & 0.05 & 0.05 & $10^{2}$ & $10^{2}$ \\
PMMA & & & & & & \\
\hline
\end{tabular}

152 To simulate acquisitions realized with this pencil chamber, integrated dose in the pencil beam is 153 estimated as :

$$
D I=\frac{E \times I \times t \times L}{\rho_{\text {air }} \times V \times 10^{-3}}(e q u .5)
$$


155 with $D /$ the integrated dose (in Gy.cm), $E$ the mean deposited energy (in eV/shower) obtained with 156 the MC simulation in the volume represented the pencil chamber, I the scanning current (in A), $t$ 157 the acquisition time (in s), $L$ the chamber length (in $\mathrm{cm}$ ), $\rho_{\text {air }}$ the air density (in $\mathrm{g} / \mathrm{cm}^{3}$ ) and $V$ the 158 chamber volume (in $\left.\mathrm{cm}^{3}\right)$.

159 Uncertainty on the estimated $D I$ is evaluated combining the contributions to the uncertainty budget 160 using the so called "sandwich law" described in the guide to the expression of uncertainty in 161 measurement (ISO/IEC Guide 98-3:2008):

$$
\begin{gathered}
u_{c}^{2}(D I)=\left(\frac{I \times t \times L}{\rho_{\text {air }} \times V \times 10^{-3}}\right)^{2} \times u^{2}(E)+\left(\frac{E \times t \times L}{\rho_{\text {air }} \times V \times 10^{-3}}\right)^{2} \times u^{2}(I) \\
+\left(\frac{E \times I \times L}{\rho_{\text {air }} \times V \times 10^{-3}}\right)^{2} \times u^{2}(t)+\left(\frac{E \times I \times t}{\rho_{\text {air }} \times V \times 10^{-3}}\right)^{2} \times u^{2}(L) \\
+\left(\frac{E \times I \times t \times L}{\rho_{\text {air }} \times V^{2} \times 10^{-3}}\right)^{2} \times u^{2}(V)(\text { equ. } 6)
\end{gathered}
$$

165 The values of the uncertainties $u(E), u(I), u(t), u(L)$ and $u(V)$ are detailled in paragraph 2.3.3.

\section{$166 \quad 2.3 .2 \quad$ Mean dose deposited in a volume}

167 For each CT acquisition with the anthropomorphic phantom, the DICOM images of the CIRS 168 phantom are converted by associating to each Hounsfield Number a MC material. This conversion 169 is done by using a calibration function determined with the CIRS Electron density phantom. The 170 calibration function is reported in figure 2. Only four materials are considered here: air, bone, soft 171 tissue and lung. 


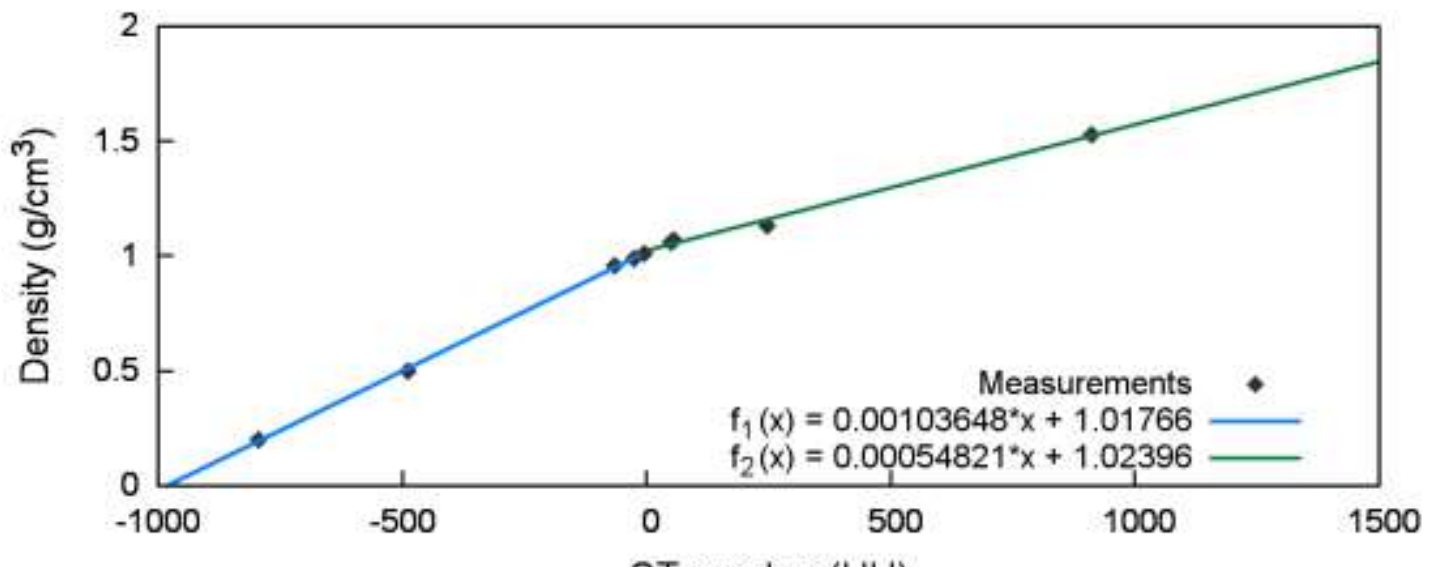

172

CT number (HU)

Figure 2: Calibration function for the DICOM images of the CIRS phantom.

174 For point doses comparison, the values obtained in the voxels corresponding to inserts containing

175 OSL are compared to measurements.

176 For dose maps comparison, the values obtained in the pixels of the slices corresponding to the

177 film position are compared to measurements. To do that, dose maps from film read-outs and from

178 MC simulations are centered. As pixel size is not the same between experimental dose map

$179(0.51 \mathrm{~mm})$ and $\mathrm{MC}$ simulations $(2.9 \mathrm{~mm})$ normalized dose profiles are plotted to compare the 180 results.

181 To improve the MC computation time for X-ray dosimetric purposes, secondary electrons are not 182 tracked if their range is smaller than the considered voxel size (Deak et al 2008 and Li et al 2011).

183 The effects of this approximation have already been investigated in detail by Chao et al 2001, who 184 showed negligible differences between incorporating and omitting secondary electrons transport 185 for diagnostic energy beams. The parameters used for the simulation respect this assumption. 186 They are reported in table 3 for each material.

187 Table 3: Parameters used for PENELOPE simulations and for each biological material used in this study.

$\begin{array}{lllllllll}\text { Material } & E_{\text {abs,e- }}(e V) & E_{a b s, p h o t o n s}(e V) & E_{a b s, e+}(e V) & C 1 & C 2 & W_{c c}(e V) & W_{C R}(e V)\end{array}$




\begin{tabular}{lccccccc}
\hline Air & $2.10^{5}$ & $10^{2}$ & $2.10^{5}$ & 0.05 & 0.05 & $10^{2}$ & $10^{2}$ \\
Lung & $2.10^{5}$ & $10^{2}$ & $2.10^{5}$ & 0.05 & 0.05 & $10^{2}$ & $10^{2}$ \\
Soft tissue & $2.10^{5}$ & $10^{2}$ & $2.10^{5}$ & 0.05 & 0.05 & $10^{2}$ & $10^{2}$ \\
Bone & $2.10^{5}$ & $10^{2}$ & $2.10^{5}$ & 0.05 & 0.05 & $10^{2}$ & $10^{2}$ \\
\hline
\end{tabular}

188 To validate the MC model in the anthropomorphic phantom, the mean dose in a voxel has to be 189 estimated as:

190

$$
D=D_{M C} \times I \times t \times 1000(e q u .7)
$$

191 with $D$ the dose in the voxel (in Gy), $D_{M C}$ the estimated MC dose value (in eV/g/shower), I the 192 scanning current (in A) and $t$ the acquisition time (in s).

193 Uncertainty on the estimated $D$ is also evaluated combining the contributions to the uncertainty

194 budget using the so called "sandwich law" described in the guide to the expression of uncertainty 195 in measurement:

$$
u_{c}^{2}(D)=u^{2}\left(D_{M C}\right) \times(\mathrm{I} \times \mathrm{t} \times 1000)^{2}+u^{2}(\mathrm{I}) \times\left(D_{M C} \times \mathrm{t} \times 1000\right)^{2}
$$

$$
+u^{2}(\mathrm{t}) \times\left(D_{M C} \times \mathrm{I} \times 1000\right)^{2}(e q u .8)
$$

198 The values of the uncertainties $u\left(D_{M C}\right), u(l)$ and $u(t)$ are detailled in paragraph 2.3.3.

\section{$199 \quad 2.3 .3 \quad$ Uncertainty budget}

200 The stochastic uncertainties $u(E)$ and $u\left(D_{M C}\right)$ due to $M C$ simulation vary for each simulation. It is 201 given with a coverage factor $k=3$. Uncertainty of the current $(u(I))$ and the acquisition time $(u(t))$ 202 are given in the technical note of the CT :

$$
u(I)=\mp(10 \%+0.5 m A)(e q u .9)
$$

$$
u(t)=\mp(5 \%+10 m s)(e q u .10)
$$


205 However, the confidence interval associated with these uncertainties is not specified. According 206 to the recommendations of the guide for the expression of measurement uncertainty, we decided 207 to consider that the uncertainties were expressed for a confidence interval of $k=1$ and that the 208 variable follows a uniform probability law. No information about $u(V)$ and $u(L)$ are given. According 209 to the accuracy needed to build such ionization chamber, we assume that these uncertainties can 210 be considered as negligeable.

211 Uncertainties are combined with the same confidence interval. In the following they are presented 212 at $\mathrm{k}=2$, ie with a confidence interval of $\approx 95 \%$.

213 It is also considered that all the variables are independent that is to say that the covariance are

214 not taken into account, this can lead to overestimate the uncertainties. It is assume that this 215 overestimate is not large.

2163 Model validation in homogeneous conditions

$217 \quad 3.1 \quad$ Isocenter validation

218 Integral of the air kerma over $100 \mathrm{~mm}$ is measured with a 10X6-3CT Radcal pencil ionization 219 chamber (figure 3a). The chamber is introduced into a home-made PMMA tube of $3 \mathrm{~cm}$ exterior 220 diameter. Acquisitions have been performed at $100 \mathrm{kVp}$ and $120 \mathrm{kVp}$, with both bowtie filters, a $22140 \mathrm{~mm}$ nominal collimation, a $50 \mathrm{~cm}$ beam length, $300 \mathrm{mAs}$ and the $\mathrm{X}$-ray tube positioned at the 222 top of the gantry (figure $3 b$ ). 


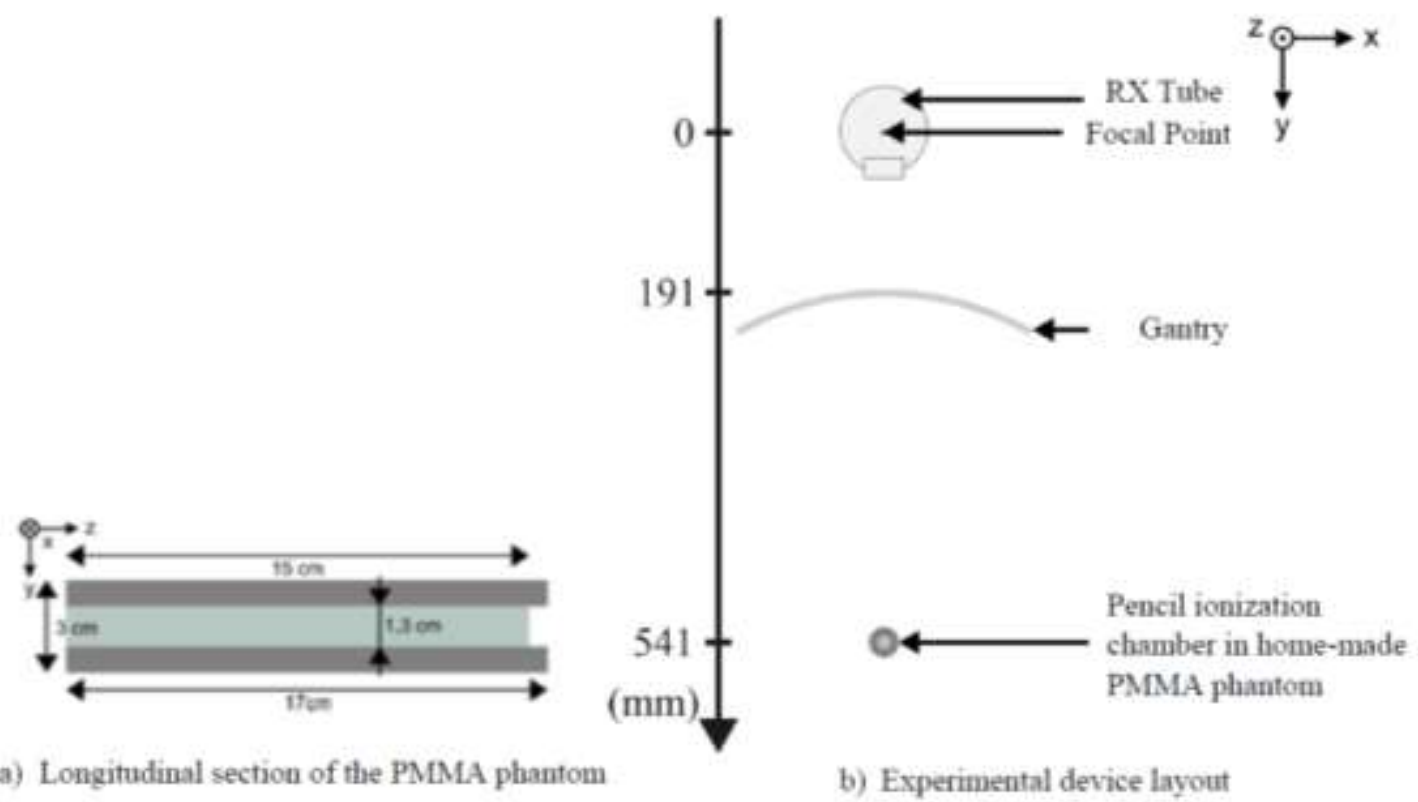

Figure 3: Longitudinal section of the PMMA phantom (a) and experimental set-up diagram (b).

$225 \quad 3.2$ "Air kerma" index validation

226 "Air kerma" index is a dose metric of the CT which represents the amount of radiations emitted 227 per rotation of the RX tube. To determine "air kerma" index, two cylindrical phantoms (figure 4) 228 composed of one central inserts and 4 peripheral inserts are used:

229 - The "head phantom" (figure 4a) has a diameter of $16 \mathrm{~cm}$. It is used to calculate the air $230 \quad$ kerma index for head and children CT acquisitions.

- The "body phantom" (figure $4 \mathrm{~b}$ ) has a diameter of $32 \mathrm{~cm}$. It is used to determine the air kerma index for body CT acquisitions

233 The air kerma index CTDIw (for Weighting Computed Tomographic Dose Index) is defined as:

$$
C T D I_{W}=\frac{1}{3}\left(C_{P M M A, 100, c}+2 \times C_{P M M A, 100, p}\right)(e q u .11)
$$


235 with $C_{P M M A, 100, c}$ being the value of the air kerma index obtained when the pencil chamber is 236 localized in the central location and $C_{P M M A, 100, p}$ being the mean value of the air kerma index when 237 the pencil chamber is inserted in the 4 peripheral locations.

238 Acquisitions have been performed at $100 \mathrm{kVp}$ and $120 \mathrm{kVp}$ and for a $40 \mathrm{~mm}$ nominal collimation.

239 The bowtie filter corresponding to the phantom has been used, thus the Ped Body filter for the 240 head phantom and the Large Body filter for the body phantom. All acquisitions have been 241 performed with the Radcal pencil chamber, $600 \mathrm{~mA}$ and an acquisition time of $1 \mathrm{~s}$. The phantom

242 is positioned at the CT isocenter. The pencil chamber is positioned in one of the insert while the 243 other inserts are filled with PMMA cylinders. Five acquisitions are needed to get the air kerma 244 index in the 5 inserts of each phantom.

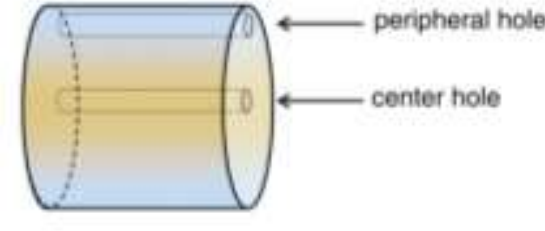

a) Head phantom

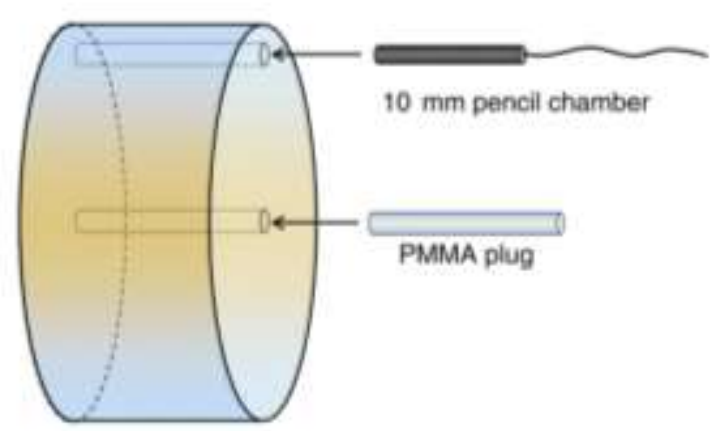

b) Body phantom

\section{$247 \quad 3.3 \quad$ Uncertainty budget}

248 Measurements uncertainties are evaluated from information reported in the AIEA report 457 about

249 the dosimetric practice in diagnostic imaging (IAEA-TRS 457). Scenario 1 has been chosen to 250 estimate the uncertainty budget. In this scenario, the device is used in conformity with the CEI 25161674 norm. According to the data reported in the AIEA report and to the fact that the pencil 252 chamber is inserted in a PPMA phantom, experimental results are presented with a relative 253 uncertainty of $13.3 \%$. 
$4 \quad$ Model validation in clinical conditions

255 Several acquisitions (table 4) are performed combining different parameters (high voltage, pitch, 256 SFOV).

257 Table 4: Acquisitions performed experimentally and with the MC simulation to estimate point dose 258 values.

\begin{tabular}{cccc}
\hline \hline $\mathrm{kVp}$ & Bowtie filter & Pitch & $\mathrm{mAs}$ \\
& & 0.984 & 570 \\
100 & Large & 1.375 & 750 \\
& & & 95 \\
\cline { 2 - 4 } 120 & Ped & 0.516 & 150 \\
\hline \hline
\end{tabular}

\subsection{CIRS anthropomorphic phantom}

260 The ATOM adult female phantom from CIRS is used to perform measurements with Optically 261 Stimulated Luminescence dosimeters (OSLDs). This phantom has slots in different localizations 262 and tissues to insert OSLDs (figure 5). In table 5 are reported the positions of the inserts in which 263 OSLDs have been inserted for both acquisitions (head and thorax).
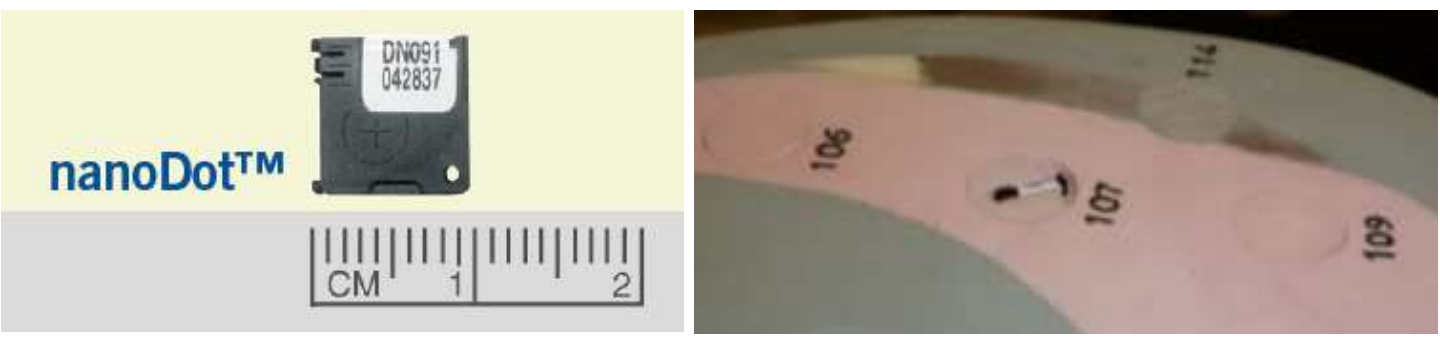

265 Figure 5: Photo of an OSLD on the left and photo of an OSLD inserted in a CIRS phantom slot on the right. 
Table 5: OSLD locations in the anthropomorphic phantom for thorax and head acquisitions.

\begin{tabular}{ccccc}
\hline \hline & Location & Tissue & Section & Position \\
\hline \multirow{2}{*}{ Thorax } & Sight Lung & Loft tissue & 21 & 137 \\
& Spine & Bone & 19 & 107 \\
& Heart & Soft tissue & 16 & 102 \\
& Rib & Bone & 15 & 83 \\
\hline \multirow{3}{*}{ Head } & Brain & Soft tissue & 3 & 69 \\
& Skull & Bone & 3 & 6 \\
& Mandible & Bone & 7 & 10 \\
\hline \hline
\end{tabular}

\section{$269 \quad 4.2 \quad \underline{\text { OSL dose assessment }}$}

270 In their review, Yukihara and McKeever 2008, show the possibility of using OSLD for CT

271 dosimetry. The great advantages of these detectors is their uniformity in sensitivity because the

$272 \mathrm{Al}_{2} \mathrm{O}_{3}: \mathrm{C}$ powder used in the production process is a homogenized mixture of different crystal

273 growth runs. The NanoDot ${ }^{\mathrm{TM}}$ (Landauer Inc.) contain single circular OSLD (5.0 mm in diameter)

274 placed in an adapter. An effective depth of $0.1 \mathrm{~g} / \mathrm{cm}^{3}$ is assumed as the point of measurement.

275 Yukihara and McKeever mention the OSLD energy dependence, showing variations between 20

276 and $30 \%$ for potentials ranging from 80 to $140 \mathrm{kVp}$. We have thus developed a specific user guide

277 to take into account this energy dependence. 


\subsubsection{Detector calibration}

279 The read-out is performed with the semi-automatic reader MicroStar $^{\mathrm{TM}}$ NanoDot $^{\mathrm{TM}}$ system. $^{2}$ 280 Depending on the dose level, two light intensities are possible: for low doses (<200 mGy) all 38 281 LEDs are used and for high doses only 6 LEDs are used. For our application, the reader is always 282 used in the low dose regime.

283 A calibration of our own OSLD has been carried out on a range from 0 to $150 \mathrm{mGy}$. Five radiation 284 qualities $\left({ }^{137} \mathrm{Cs}\right.$ source, ${ }^{60} \mathrm{Co}$ source, RQR 4, RQR 6 and RQR 9 (NF EN 61267 Norm)), available 285 at the French national metrological laboratory (Laboratoire national Henri Becquerel, LNHB), are 286 used. Five detectors have been irradiated for each air kerma value. Average OSLD readings are 287 used to calculate the calibration factor for each beam quality, assuming that OSLD response is 288 linear in terms of air kerma. Results are reported in table 6.

289 Table 6: Calibration function (OSLD reading as a function of air kerma) obtained for the different beam 290 qualities.

\begin{tabular}{cc}
\hline Beam quality & Calibration function \\
\hline RQR 4 & $R=6781.4 \times K_{a}+2475.5$ \\
RQR 6 & $R=6210.6 \times K_{a}+2528.5$ \\
RQR 9 & $R=5551.5 \times K_{a}+2647.1$ \\
${ }^{137}$ Cs & $R=2007.6 \times K_{a}+2573.1$ \\
${ }^{60}$ Co & $R=1949.6 \times K_{a}+2531.6$ \\
\hline
\end{tabular}

\subsubsection{Detector read-out analysis}

292 Irradiated OSLD, used for measurement, as well as non-irradiated OSLD, are read three times. 293 Mean reading values for irradiated $\left(\bar{r}_{I}\right)$ and non-irradiated OSLD $\left(\overline{r_{N I}}\right)$ are then computed. These 294 mean raw reading values are then corrected individually from the OSLD sensitivity (se) to obtain 
295 the real reading value for irradiated $\left(\overline{R_{I}}\right)$ and non-irradiated $\left(\overline{R_{N I}}\right)$ OSLD, respectively. Finally, the

296 corrected signal S, used to determine the dose absorbed by the OSLD is calculated by subtracting 297 raw reading values for irradiated $\left(\overline{R_{I}}\right)$ and non-irradiated $\left(\overline{R_{N I}}\right)$ OSLD.

298 To take into account the energy dependence of the OSL detectors, protocol detailed in 299 Bordy et al 2013 is adapted. The energy spectrum corresponding to the OSL position is 300 determined by MC simulation. Calibration factors for each energy bins are convolved using the 301 energy spectrum as weight to adapt the calibration factor to the spectrum at the point of 302 measurement. Assuming the electronic equilibrium condition are fulfilled, the dose in the tissue is 303 obtained by multiplying the air kerma by the ratio of the interaction coefficients:

304

$$
D_{\text {medium } m, Q}=K_{\text {air }, Q 0} \times\left(\frac{\mu}{\rho}\right)_{Q 0, \text { air }}^{Q, m}(\text { equ.12) }
$$

305 with $\left(\frac{\mu}{\rho}\right)_{Q 0, \text { air }}^{Q, m}={ }^{\left(\frac{\mu_{e n}}{\rho}\right)_{Q, m} /\left(\frac{\mu_{t r}}{\rho}\right)_{Q 0, a i r}}$

\section{$306 \quad 4.2 .3 \quad$ Uncertainty budget}

307 For each step of the OSLD read-out an uncertainty budget is calculated. At the end, the uncertainty 308 associated to the absorbed dose $(D)$ in the medium is defined as:

309

$$
U_{D}^{2}=U_{K}^{2}\left[\left(\frac{\mu}{\rho}\right)_{Q 0, a i r}^{Q, m}\right]^{2}+U_{\left(\mu_{e n} / \rho\right) Q, m}^{2}\left[\frac{K}{\left(\frac{\mu_{t r}}{\rho}\right)_{Q 0, a i r}}\right]^{2}+U_{\left(\mu_{t r} / \rho\right) Q 0, a i r}^{2}\left[\frac{K \times\left(\frac{\mu_{e n}}{\rho}\right)_{Q, m}}{\left(\frac{\mu_{t r}}{\rho}\right)_{Q 0, a i r}}\right]^{2}(\text { equ.13) }
$$

310 with $U_{K}$ the uncertainty associated to the air kerma and defined as :

$$
U_{K}=\left(\frac{1}{a_{Q}}\right)^{2} \times U_{S}^{2}+\left(\frac{-S}{a_{Q}^{2}}\right)^{2} \times U_{a Q}^{2}(\text { equ. } 14)
$$


312 where $U_{a Q}$ is the uncertainty associated to $a_{Q}$ the slope of the calibration function corresponding 313 to the energy spectrum observed at the point of measurement and defined as :

$$
U_{a q}^{2}=\left(\frac{1}{\sum_{i} N_{i} \times E_{i}}\right)^{2} \sum_{i}\left(N_{i} \times E_{i} \times U_{a(E i)}\right)^{2}(e q u .15)
$$

315 and $U_{S}$ is the uncertainty associated to the OSLD read-out :

$$
U_{S}^{2}=U_{\bar{R}_{I}}^{2}+U_{\bar{R}_{N I}}^{2}(e q u .16)
$$

317 with $U_{\bar{R}_{I}}$ being the uncertainty associated to the irradiated detectors and $U_{\bar{R}_{N I}}$ being the uncertainty

318 associated to the non-irradiated detectors ; both of them are calculated thanks to the following 319 equation :

$$
U_{\bar{R}}^{2}=\left(\frac{1}{s e}\right)^{2} \times U+\left(\frac{U_{\bar{r}}}{s e \times s e}\right)^{2} \times U_{s e}^{2}(e q u .17)
$$

321 The uncertainty on the mass interaction coefficients is taken equal to $1 \%$, according to the NIST

322 (https://www.nist.gov/).

\section{$323 \quad 4.3$ Dose maps assessment with XR-QA films}

324 When exposed to radiation, the organic based dye of radiochromic films changes color due to 325 polymerization: the color of XR-QA films turns from orange to brownish-black depending on the 326 level of exposure (see figure 6). Several features of these detectors have attracted the attention 327 of the medical physcis community: insensitivity to visible light, self-developing characteristics, 328 dose-rate independence. 


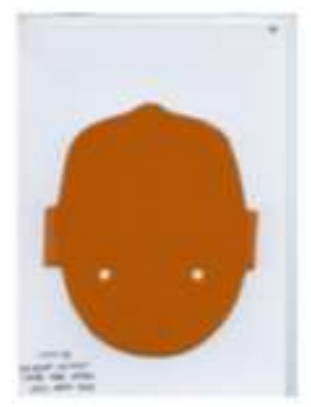

(a) Before irradiation

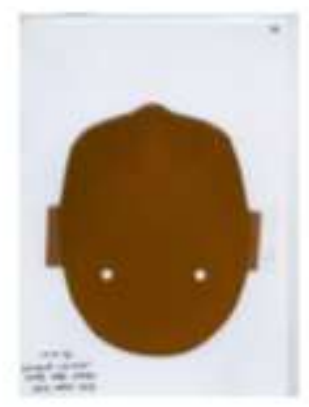

(b) After irradiation

Figure 6: Film read-out for the head acquisition: the color of XR-QA films turns from orange to

332 Rampodo et al 2006 studied the dependence of XR-QA films for kilovolt energies and they

333 proposed a method to use these films (reading, calibration, uncertainties assessment). They also

334 highlighted a variation of the film response with beam energy which can go up to $20 \%$. Boivin et

335 al 2011 proposed also to use the films for in vivo dosimetry purposes in medical imaging. More

336 recently, Tomic et al 2014 proposed a method for calibrating and correcting the film reading. They

337 showed that the use of a single calibration function leads to a relative uncertainty of $14 \%$ on the

338 dose values, if the calibration function is obtained for a beam quality taken in the middle of the

339 investigated energy range. In the following we have considered this value for the uncertainty

340 associated to the film analysis.

\section{$341 \quad$ 4.3.1 Film calibration}

342 The films calibration has been carried out in the LM2S (Laboratoire Modélisation, Systèmes et

343 Simulation) laboratory for a $120 \mathrm{kVp}$ X-ray beam with a HVL of $7.14 \mathrm{~mm}$ aluminum. Films and a

344 Farmer 30013 PTW ionization chamber have been irradiated at the same time in order to

345 determine the air kerma associated to the film read-out. The Farmer chamber has been previously

346 calibrated at the French national laboratory of metrology (LNHB) in terms of air kerma. Films are

347 read before and one week after irradiation and saved as TIF files. Unirradiated film reading is also 
348 necessary to obtain net optical density. It is also recommended to control time between irradiation

349 and read-out (at least $24 \mathrm{~h})$. By using only the red channel, the mean pixel values before $\left(P V_{N I}\right)$

350 and after $\left(P V_{I}\right)$ irradiation are calculated in a mean $1 \mathrm{~mm}^{2}$ region of interest. The net optical density

351 is then defined in equation 17 :

352

$$
n e t O D=\log _{10}\left(\frac{P V_{N I}}{P V_{I}}\right)(e q u .18)
$$

353 The calibration function linking the air kerma and the netOD has been adjusted according the 354 following polynomial function (equation 18):

$$
K_{\text {air }, Q 0}=a \times n e t O D+b \times n e t O D^{4}(e q u .19)
$$

356 As for OSLD the dose in the tissue is obtained by multiplying the air kerma by the ratio of the mass 357 interaction coefficients.

\section{$358 \quad 4.3 .2 \quad$ Film analysis}

359 Gafchromic XR-QA2 films are irradiated to study the dose gradient. Films are cut to fit the 360 anatomical shapes of the female anthropomorphic phantom and placed between two phantom 361 slices. For thorax acquisitions, films cannot be inserted into the breasts of the phantom because 362 breast are made from a single piece without any insert or slice. Stencil of the films contours are 363 used to ensure the reproducibility of the film positioning during the reading steps before and after 364 irradiation. For all acquisitions, the tube speed is fixed at $0.7 \mathrm{~s} / \mathrm{rot}$ and the films are read four times

365 before and one week after the irradiation. Films are read several times to ensure that film storage 366 and handling have been performed in good conditions (dry and dark environment, no dust or 367 fingerprints ..). Optical density values are then converted into air kerma according to the calibration 368 function.

369 A thoracic and a head acquisition (table 7) have been performed.

370 Table 7: Acquisitions performed experimentally and with the MC simulation to estimate dose maps. 


\section{kVp Bowtie filter Pitch Position in the phantom}

$100 \quad$ Large $\quad 0.516 \quad$ Between section 16 and 17

$120 \quad$ Ped $\quad 1.375 \quad$ Between section 7 and 8

\section{Organ dose estimation}

372 Organ doses for a thoracic localisation have then been estimated into the female phantom

373 provided in ICRP Publication 110. Simulations are performed for the Large Body bowtie filter with

374 a $50 \mathrm{~cm}$ SFOV, 100 and $120 \mathrm{kVp}$, a $40 \mathrm{~mm}$ collimation adapted to the used focal spot (42.6 and

$37542.9 \mathrm{~mm})$, three $\mathrm{mAs}(100,200$ and 300$)$, a $0.7 \mathrm{~s} /$ rot tube speed and three pitch values $(0.531-$

$3760.969-1.375)$. Dose values to each voxel across all the voxels belonging to each organ are

377 averaged. According to the organ $\mathrm{w}_{\mathrm{T}}$ factors reported in the ICRP 103, we decid to report the dose

378 absorbed by the more sensitive organs thus the left breast glandular tissue, the stomach wall, the

379 left pulmonary tissue, the esophagus and the spinal cord.

3806 Comparison of experimental and simulated results

381 To compare experimental results $\left(r_{\text {exp }}, \sigma_{\exp }\right)$ and $M C$ estimation $\left(r_{M C}, \sigma_{M C}\right)$ we have used 2 index :

382 - the deviation defined as :

$$
\operatorname{dev}=\frac{r_{M C}-r_{\text {exp }}}{r_{\text {exp }}} \times 100(e q u .21)
$$

$384 \quad-\quad$ and the overlap defined as : 


$$
o v l p=100 \times e^{-\frac{\left(r_{M C}-r_{e x p}\right)^{2}}{2\left(\sigma_{r M C}^{2}+\sigma_{\text {exp }}^{2}\right)}}(e q u .22)
$$

\section{$7 \quad$ Results}

\subsection{Isocenter experimental validation}

388 Table 8 shows the measured and simulated integral of the air kerma over $100 \mathrm{~mm}$. Results show 389 a good agreement between the simulations and the measurements with a deviation less than $10 \%$ 390 and an overlap larger than $87 \%$ for the 4 cases considered here.

Table 8: Measured and simulated values obtained for the integral of the air kerma in the pencil chamber.

\begin{tabular}{lccccc}
\hline \hline & & MC estimation & Experimental value & \multicolumn{2}{c}{ Comparison } \\
& & \multicolumn{2}{c}{$D I \pm U_{D I}(\mathrm{mGy} . \mathrm{cm})$} & $\operatorname{dev}(\%)$ & ovlp (\%) \\
\hline $\begin{array}{l}\text { Ped Body } \\
\text { Bowie filter }\end{array}$ & $100 \mathrm{kV}$ & $289.9 \pm 35.1$ & $278.6 \pm 18.4$ & 4.0 & 96.0 \\
& $120 \mathrm{kV}$ & $400.6 \pm 48.4$ & $408.2 \pm 26.9$ & -1.9 & 99.1 \\
$\begin{array}{l}\text { Large Body } \\
\text { Bowie filter }\end{array}$ & $100 \mathrm{kV}$ & $206.7 \pm 25.5$ & $216.1 \pm 14.3$ & -4.4 & 94.9 \\
& $120 \mathrm{kV}$ & $309.1 \pm 37.8$ & $331.4 \pm 21.9$ & -6.7 & 87.8 \\
\hline \hline
\end{tabular}

395 Table 9 shows the measured and simulated integral of the air kerma index obtained with both 396 phantoms. Results show a good agreement between the simulations and the measurements with 397 a deviation less than $4 \%$ and an overlap larger than $89 \%$ for the 4 cases considered here. 


\begin{tabular}{lccccc}
\hline \hline & & MC estimation & Experimental value & \multicolumn{2}{c}{ Comparison } \\
& & \multicolumn{2}{c}{$C T D I_{w} \pm U_{C w}(\mathrm{mGy})$} & $\operatorname{dev}(\%)$ & ovlp (\%) \\
\hline $\begin{array}{l}\text { Ped Body } \\
\text { Bowie filter }\end{array}$ & $100 \mathrm{kV}$ & $76.3 \pm 4.4$ & $74.2 \pm 4.9$ & 2.8 & 95.0 \\
& $120 \mathrm{kV}$ & $112.9 \pm 6.4$ & $117.6 \pm 7.8$ & -4.0 & 89.4 \\
Large Body & $100 \mathrm{kV}$ & $37.7 \pm 2.6$ & $36.5 \pm 2.4$ & 3.2 & 94.7 \\
Bowie filter & $120 \mathrm{kV}$ & $58.7 \pm 4.0$ & $59.0 \pm 3.9$ & -0.5 & 99.8 \\
\hline \hline
\end{tabular}

403

$404 \quad 7.3$ Point dose comparison

405 Experimental and simulated dose values are reported in figure 7 as well as the relation between 406 them:

$$
D_{\text {exp }}=0.867 \times D_{\text {simul }}(\text { equ.23) }
$$

408 Simulated values are on average higher than the experimental ones. The uncertainty bars plotted 409 in figure 7 is obtained by fitting the MC uncertainty associated to each simulated values. This one 410 is about $23.2 \%(k=2)$ for all the simulations performed here. The experimental uncertainty is about $411 \quad 7.4 \%$. 


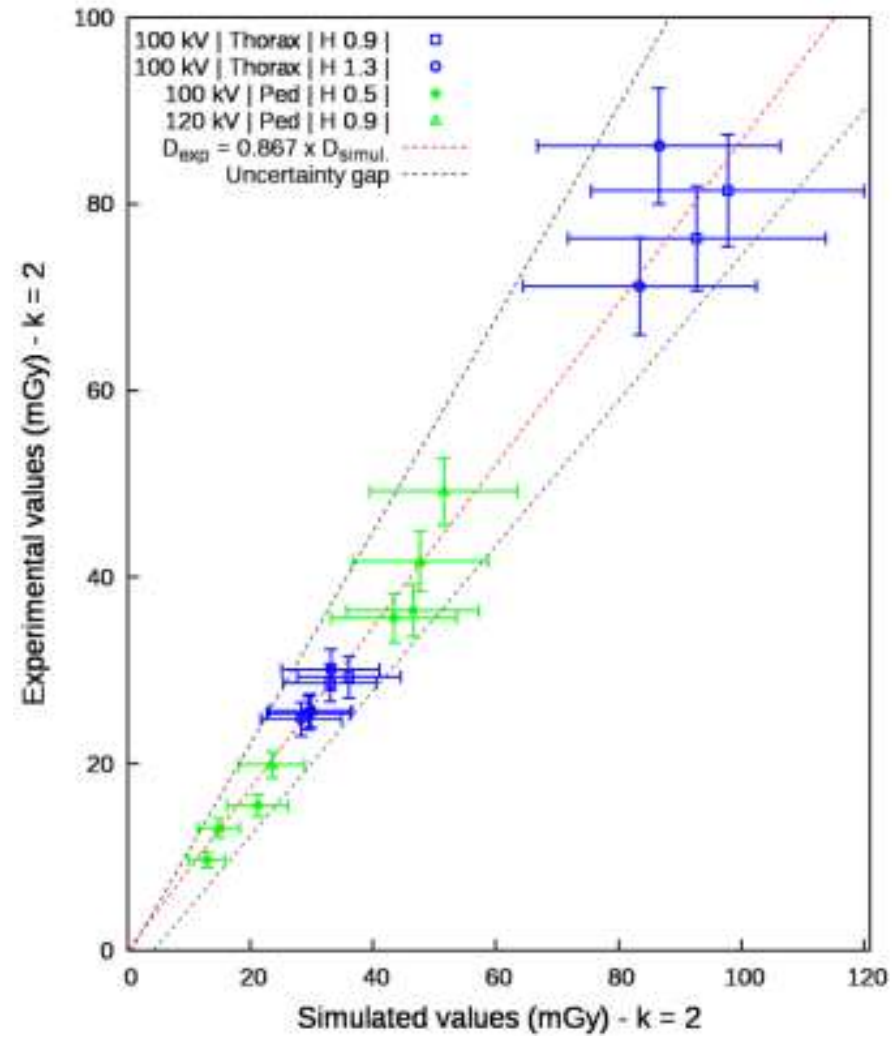

Figure 7: Experimental dose values versus simulated dose values and uncertainties given at $k=2$ for the head (in green) and thorax (in blue) acquisitions with the female ATOM phantom. The relation between both dose values are fitted (in red) as well as the uncertainty gap (in black).

\subsection{Dose maps comparison}

417 Experimental and simulated dose distributions for a head scan are reported in figure 8. Large 418 differences can be observed between the two maps and will be discussed later in the discussion section. 

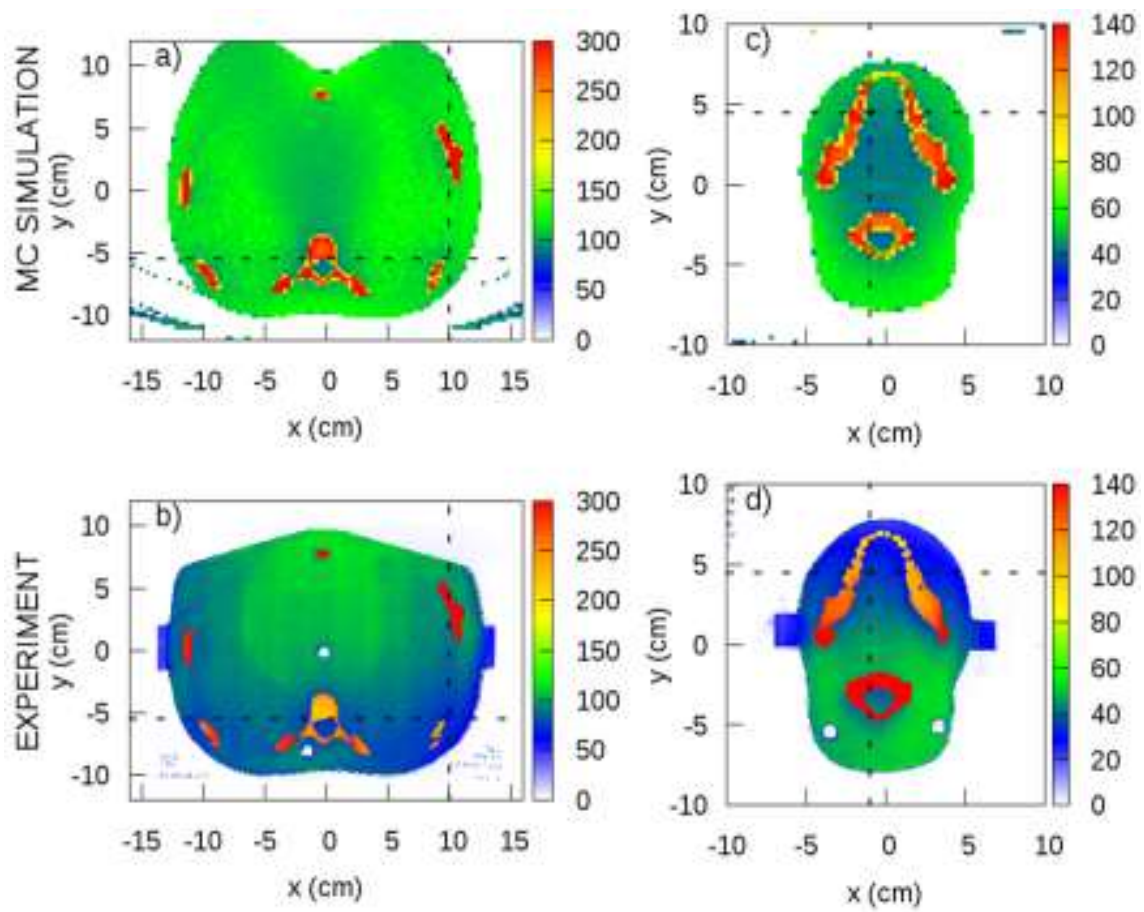

Figure 8: Simulated and measured dose distributions in mGy for a head (c and d) and a thorax ( $a$ and $b$ ) scan. Dashed lines indicate the profiles used for figure 9.

Validation of the dose gradient is performed by comparing simulated and experimental profiles

425 reported in figure 9 and obtained from figures $8 a, 8 b, 8 c$ and $8 d$. In figures 8 and 9 the $M C$ 426 uncertainty is about $3 \%$ and the experimental uncertainty about $15 \%$. Deviations between 427 simulations and experiments are less than $20 \%$ in bone heterogeneities. In soft tissue regions the 428 deviation can be higher, especially for the head acquisition. This deviation is mainly due to the 429 path of the x-ray tube during the experimental scan which is not simulated accurately in the MC 430 simulation, the initial position of the tube being experimentally unavailable. 

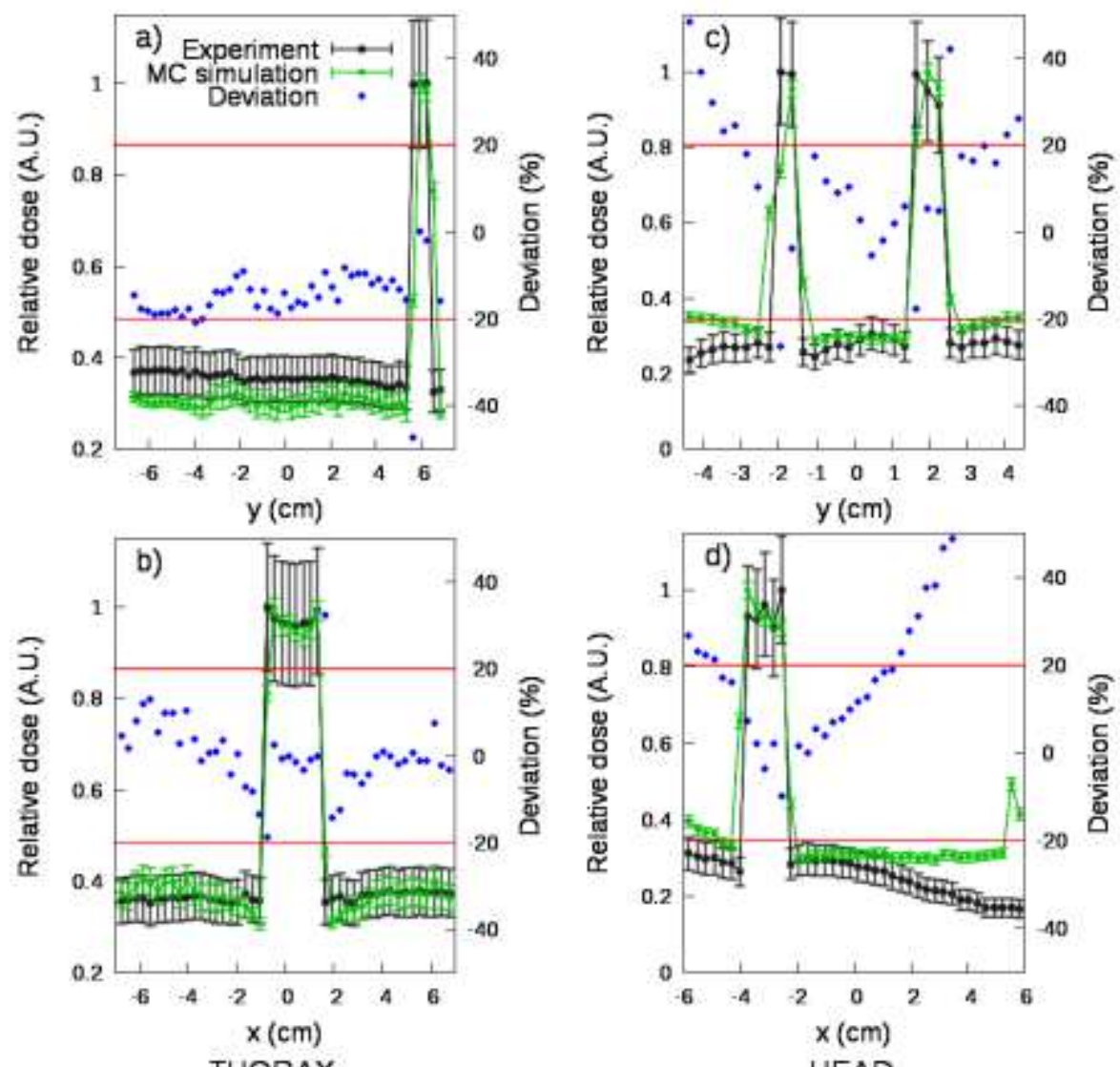

THORAX

HEAD

432 Figure 9: Simulated and measured relative dose profiles for the head ( $c$ and d) and the thorax ( $a$ and $b$ ) acquisitions. The profiles are obtained for the dashed lines reported in figure 8 . The deviation between simulation and measurement is reported in blue.

\subsection{Organ dose estimation}

436 Organ doses for the Large Body Bowtie filter, $120 \mathrm{kVp}, 40 \mathrm{~mm}$ collimation, 1.375 pitch and

$437100 \mathrm{mAs}$, are reported in table 10 and compared with Zhang et al 2012 results, who also estimated

438 organ dose for the female phantom described in the ICRP 110. They simulated in details the GE

439 VCT LightSpeed 64 thanks to accurate data provided by the manufacturer. They used a modified

440 version of PENELOPE reported in Li et al 2011 to estimate organ doses in the ICRP 110 phantoms.

441 Our results, obtained in less than one hour in $24 \mathrm{CPU}$ for all cases, are a little bit larger than the

442 dose values reported by Zhang et al 2012. Deviations are less than $5.8 \%$ for the four organs

443 considered here. 


\begin{tabular}{|c|c|c|c|}
\hline \multicolumn{4}{|c|}{ Simulated organ doses (mGy) } \\
\hline Organs & & & Deviations(\%) \\
\hline & This study & Zhang et al 2012 & \\
\hline Esophagus & $9.8(0.003)$ & 9.3 & 5.4 \\
\hline Lung & $10.9(0.003)$ & 10.3 & 5.8 \\
\hline Breast & $10.2(0.002)$ & 10.1 & 1.0 \\
\hline Stomach & $11.1(0.001)$ & 10.9 & 1.8 \\
\hline
\end{tabular}

\section{Discussion and Conclusion}

448 Despite a lack of information about the scanner geometry, the GE VCT LightSpeed 64 has been

449 modelled by adapting the method developed by Turner et al 2009.

450 Results obtained in homogeneous conditions validate the use of the MC model for dosimetric 451 estimation. Measured and simulated integrals of the air kerma over $100 \mathrm{~mm}$ are in agreement;

452 this also validates the use of the tube load information to convert simulated results into Gray. By 453 comparing integral of the air kerma in table 8 and their associated uncertainties, we note that the 454 simulation uncertainties budget is actually deteriorated by the conversion factor contribution. 455 According to the manufacturer technical note, current and acquisition time have $10 \%$ and $5 \%$ 456 uncertainty on the displayed value, respectively. Despite these values, relative uncertainties are 457 below $15 \%$. Such uncertainties are compatible with medical imaging applications. 
458 Measured and simulated point dose obtained in anthropomorphic conditions show deviations up 459 to $15 \%$. However confidence intervals are overlapped allowing us to conclude that results are in 460 agreement. The uncertainty budget for simulated doses is mainly by the conversion factor 461 uncertainties. The relative uncertainties for the tube current and the acquisition time are 462 respectively $10 \%$ and $5 \%$ at $k=1$, as reported in the technical note. Simulated dose uncertainties 463 might seem quite large (about $22 \%$ at $k=2$ ), but such uncertainties are compatible with dosimetric 464 purposes in medical imaging. The benefit of the conversion factor is therefore maintained.

465 Large differences in the simulated and the experimental dose distributions can be noted. All the 466 experimental dose maps show an important effect of the initial tube position, as already reported 467 by several authors (Li et al 2011 and Zhang et al 2009). The surface dose distribution resulting 468 from a helical acquisition is periodic (Dixon and Ballard 2007) and the tube start angle determines 469 the location of the high and low dose regions. It has been reported by (Zhang et al 2009) that the 470 magnitude of organ dose reduction resulting from varying tube start angle varies from 10 to $30 \%$ 471 depending on the location and size of the organs. In the experimental dose distribution, shown in 472 figures $8 \mathrm{~b}$ and $8 \mathrm{~d}$, the tube position relative to the phantom slice containing the film can be easily 473 determined, since a higher dose is delivered to the top and the back of the phantom for the thorax 474 and the head acquisition, respectively. However, the tube path relative to the patient cannot be 475 fully worked out because the tube starting angle information is not provided on the GE VCT 476 Lightspeed 64 scanner. Taking into account this lack of information it has been decided to 477 randomly sample the tube starting angle for each simulated particle. By making this choice, all 478 possible tube paths are simulated, leading to a more homogenous dose distribution (figures 8a 479 and 8c) and an overestimation for some location of the real delivered dose. Nevertheless, instead 480 of underestimating the dose for radiosensitive organs the MC simulation considers the worst case 481 and provides the maximum dose which could be delivered. 
482 For directly irradiated areas there are sometimes some differences in the vicinity of bone 483 structures. They are mainly due to the different pixel sizes between the film and the dose matrices.

484 Due to the small number of particles, the size of the voxels in the dose matrix cannot match the 485 size of that of the film. Indeed, the smaller the size of the voxels, the more one has to increase the 486 number of particles to converge the simulation.

487 In addition to the difficulties related to the difference in resolution, the dose maps from the 488 simulation highlight problems related to voxelization of the phantom. Since the voxels are larger 489 than those used for the phantom, a voxel in the dose grid can be composed of several tissues 490 (lung, bone and soft tissue in our case). If a voxel is composed of several tissues, it can be 491 considered as being composed of an hybrid tissue associated with an intermediate density 492 according to the densities of the materials initially present and their density. The dose deposit is 493 then affected and the separation between the tissues is less marked. However, the dose profiles 494 show that the gradients are still well respected.

495 Besides, in their article Long et al 2013 showed from MC simulations that the starting angle could 496 lead to organ dose differences between $-20 \%$ and $34 \%$ compared to the average value. We also 497 found such discrepancies when comparing simulations and measurements obtained with OSLD. 498 By combining the information of Long's article and the non-homogeneous dose deposit visible on 499 the films due to the random draw of the starting position, the important differences found in the 500 comparison between measurement and simulation for the OSLDs can be explained.

501 For one studied case, organ dose estimations with our software are in agreement with those 502 published by Zhang et al 2012, attesting the reliability of the developed software. Organ dose 503 estimation in the ICRP 110 phantoms can be thus performed in a short notice, less than one hour 504 using $24 \mathrm{CPU}$. In the future, improvements would be considered to reduce the simulation time. 


\section{Acknowledgments}

507 The authors thank warmly Helena Chesneau for the calibration of the Gafchromic films and Fabien

508 Moignau, Marc Denoziere, Nelly Lecerf, for their help in the calibration of the OSLD.

\section{References}

510 Akhlaghi P., Miri-Hakimabad H. and Rafat-Motavalli L. (2015) Dose estimation in reference and

511 non-reference pediatric patients undergoing computed tomography examinations: a Monte Carlo

512 study, Radioprotection 50, 43-54.

513 Alaei P., Gerbi B. J. and Geise R. A. (2000) Evaluation of a model-based treatment planning

514 system for dose computations in the kilovoltage energy range, Med. Phys. 27, 2821-2826.

515 Amis E. S. (2011) CT Radiation Dose; Trending in the Right Direction, Radiology 261, 5-8.

516 Boivin J., Tomic N., Fadallah B., DeBlois F., and Devic S. (2011) Reference dosimetry during

517 diagnostic CT examination using XR-QA radiochromic film model, Med. Phys. 38, 5119-5129.

518 Bordy J.M., Bessieres I., d'Agostino E., Domingo C., d’Errico F., di Fulvio A., Knezevic Z., Miljanic

519 S., Olko P., Ostrowsky A., Poumarede B., Sorel S., Stolarczyk L., Vermesse D.(2013)

520 Radiotherapy out-of-field dosimetry: experimental and computational results for photons in a water

521 tank Radiat. Meas. 57, 29-34.

522 Chao T. C., Bozkurt A., and Xu X. G. (2001) Conversion coefficients based on the VIP-Man 523 anatomical model and EGS4, Health Phys. 81, 163-183.

524 Coakley F.V., Gould R., Yeh B. M., and Arenson R.L. (2010) CT Radiation Dose: What can you 525 do right now in your practice?, AJR 196, 619-625. 
526 Deak P., van Straten M., Shrimpton P.C., Zankl M. and Kalender W.A. (2008) Validation of a Monte

527 Carlo tool for patient specific dose simulations in multi-slice computed tomography, Eur. Radiol.

528 18, 759-772.

529 De Marco J.J., Cagnon C.H., Cody D.D., Stevens D.M., Mc Collough C.H., O’Daniel J. and McNitt530 Gray M.F. (2005) A Monte Carlo based method to estimate radiation dose from multidetector CT 531 (MDCT): Cylindrical and anthropomorphic phantoms, Phys. Med. Biol. 50, 3989-4004.

532 Dixon R.L. and Ballard A.C. (2007) Experimental validation of a versatile system of CT dosimetry 533 using a conventional ion chamber: Beyond CTDI [sub 100] Med. Phys. 34, 3399-3413.

534 IAEA. Technical Reports Series n 457 (2007) Dosimetry in Daignostic Radiology: An International 535 Code of Practice.

536 ICRP Publication 103 (2007) The 2007 recommendations of the International Commission on 537 Radiological Protection, Ann. ICRP 37, 1-332, Pergamon Press.

538 ICRP Publication 110 (2009) Adult Reference Computational Phantoms, Ann. ICRP 39, 1-162, 539 Elsevier.

540 ISO/IEC Guide 98-3 (2008) Uncertainty of measurement - Part 3: Guide to the expression of 541 uncertainty in measurement (GUM:1995), Joint Committee for Guides in Metrology, JCGM 100.

542 Habib Geryes B., Hornbeck A., Jarrige V., Pierrat N., Ducou Le Pointe H., Dreuil S. (2019) Patient 543 dose evaluation in computed tomography: A French national study based on clinical indications, 544 Physica Medica 61,18-27.

545 Hubbell J.H. and Seltzer S.M. (2009) Tables of x-ray mass attenuation coefficients and mass 546 energy-absorption coefficients from $1 \mathrm{kev}$ to $20 \mathrm{Mev}$ for elements $Z=1$ to 92 and 48 additional 547 substances of dosimetric interest, available at: http://www.nist.gov/pml/data/xraycoef/index.cfm 
548 Jarry G., DeMarco J.J., Beifuss U., Cagnon C.H. and McNittGray M.F. (2003) A Monte Carlo based

549 method to estimate radiation dose from spiral CT: from phantom testing to patient specific models, 550 Phys. Med. Biol. 48, 2645-2663.

551 Journy N.M.Y., Lee C., Harbron R. W., McHugh K., Pearce M. S. and Berrington de Gonzales A. 552 (2017a) Projected cancer risks potentially related to past, current, and future practices in 553 paedriatric CT in the United Kingdom 1990-2020, British Journal of Cancer 116, 109-116.

554 Journy N. M. Y., Dreuil S., Boddaert N., Chateil J.-F., Defez D., Ducou-le-Pointe H., Garcier J.-M.,

555 Guersen J., Habib Geryes B., Jahnen A., Lee C., Payen-de-la-Garanderie J., Pracros J.-P., 556 Sirinelli D., Thierry-chef I. and Bernier M.-O. (2017b) Individual radiation exposure from computed 557 tomography: a survey of paediatric practice in French university hospitals, 2010-2013, European

558 Radiology 28, 1432-1084

559 Kalender W.A., Schmidt B., Zankl M. and Schmidt M. (1999) A PC program for estimating organ 560 dose and effective dose values in computed tomography Eur. Radiol. 9, 555 - 562.

561 Kalender W.A. (2014) Dose in x-ray computed tomography, Phys. Med. Biol. 59, R129-R150.

562 Li X., Segars w.P., Surgeaon G.M., Colsher J.G., Toncheva G., Yoshizumi T.T. and Frush D.P. 563 (2011) Patient-specific radiation dose and cancer risk estimation in CT: Part I. Development and 564 validation of a Monte Carlo program, Med. Phys. 38, 397 - 407.

565 Long D.I.J., Lee C., Tien C., Fisher R., Hoerner M.R., Hintenlang D. and Boch W. E. (2013) Monte 566 Carlo simulations of adult and pediatric Computed Tomography exams: Validation studies of organ 567 doses with physical phantoms, Med. Phys. 40, 013901-1-013901-10.

568 Mayo-Smith W.W., Hara A.K., Mahesh M., Sahani D.V., and Pavlicek W. (2014) How I Do It: 569 Managing Radiation Dose in CT, Radiology 273, 657-672. 
570 McCollough C.H., Leng S., Yu L., Cody D.D., Boone J.M., McNitt-Gray M.F. (2011) CT Dose Index

571 and Patient Dose: They are not the same thing, Radiology 259, 311-316.

572 Rampado O., Garelli E., Deagostini S., and Ropolo R. (2006) Dose and energy dependence of 573 response of gafchromic XR-QA film for kilovoltage x-ray beams, Phys. Med. Biol. 51, 2871-2881.

574 Sechopoulos I., Ali E.S.M., Badal A., Badano A., Boone J.M., Kyprianou I., Mainegra-Hing E., 575 McNitt-Gray M.F., McMillan K.L., Rogers D.W.O., Samei E. and Turner A.C. (2015) Monte Carlo 576 Reference Data Sets for Imaging Research, The Report of AAPM Task Group 195.

577 Sempau J., Fernandez-Varea J.M., Acosta E., and Salvat F. (2003) Experimental benchmarks of 578 the Monte Carlo code PENELOPE, Nucl. Instrum. Methods B 207, 107-123.

579 Sodickson S. (2012) Strategies for reducing radiation exposure in multi-detector row CT, Radiol. 580 Clin. N. Am. 50, 1-14.

581 Tomic N., Quintero C., Whiting B.R., Aldelaijan S., Bekerat H., Liang L., DeBlois F., Seuntjens J., 582 and Devic S. (2014) Characterization of calibration curves and energy dependence gafchromictm 583 xr-qa2 model based radiochromic film dosimetry system, Med. Phys. 41, 062105.

584 Turner A.C., Zhang D., Kim H.J., De Marco J.J., Cagnon C.H., Angel E., Cody D.D., Stevens D.M., 585 Primark A.N., McCollough C.H., McNitt-Gray M.F. (2009) A method to generate equivalent energy 586 spectra and filtration models based on measurement for multidetector CT Monte Carlo dosimetry 587 simulations, Med. Phys. 36, 2154-2164.

588 Yukihara E.G. and McKeever S.W.S. (2008) Optically stimulated luminescence (osl) dosimetry in 589 medicine, Phys. Med. Biol. 53, R351-R379. 
590 Zhang D., Zankl M., DeMarco J.J., Cagnon C.H., Angel E., Turner A.C. and McNitt-Gray M.F. 591 (2009) Reducing radiation dose to selected organs by selecting the tube start angle in MDCT 592 helical scans: A Monte Carlo based study, Med. Phys. 36, 5654-5664.

593 Zhang Y., Li X., Segars W., and Samei E. (2012) Organ doses, effective doses, and risk indices 594 in adult CT: Comparison of four types of reference phantoms across different examination 595 protocols, Med. Phys. 36, 3404-3423.

596

597

598

599

600

601

602

603

604 